\title{
On regular interstices and selective types in countable arithmetically saturated models of Peano Arithmetic
}

\author{
by \\ Teresa Bigor aj ska (Siedlce), Henryk Kot lar ski (Siedlce) \\ and James H. S ch mer l (Storrs, Conn.)
}

\begin{abstract}
We continue the earlier research of [1]. In particular, we work out a class of regular interstices and show that selective types are realized in regular interstices. We also show that, contrary to the situation above definable elements, the stabilizer of an element inside $M(0)$ whose type is selective need not be maximal.
\end{abstract}

1. Introduction. Automorphisms of a countable recursively saturated model $M$ of PA (i.e., Peano Arithmetic) were studied for many years (cf. [14] for a survey). In particular, in [10], following a remark in [8], we studied the connection between maximality of a basic open subgroup $G_{a}$ of $G=\operatorname{Aut}(M)$ and the type of $a$. It turned out that if $a$ is greater than all definable elements of $M$ then $G_{a}$ is maximal iff $\operatorname{tp}(a)$ is selective, i.e., the Skolem closure of $a$ is a minimal extension of the Skolem closure of the empty set. The goal of this paper is to investigate the situation for $a$ below some definable element (of course, under the assumption that $M$ is a model of some false completion of PA). In fact, in the main part of this paper we work with an arithmetically saturated model of PA with nonstandard definable elements.

We repeat some of the material from earlier papers for the sake of completeness. All proofs which are omitted may be found in [1].

In the main part of this paper $M$ is a countable arithmetically saturated model of PA with nonstandard definable elements. Arithmetical saturation of $M$ is equivalent to recursive saturation and $\mathbb{N}$ being strong in $M$ (cf. [11] for more in this direction).

1991 Mathematics Subject Classification: Primary 03H15; Secondary 03C62.

Research of H. Kotlarski was supported in part by The State Committee for Scientific Research (Poland), KBN, grant number 2 P03A 07110. 
For $a \in M$ we let $M(a)=$ the smallest elementary cut of $M$ which contains $a$. We also let $M[a]$ denote the greatest elementary cut of $M$ which does not contain $a$ (see [13] or [10] for more in this direction). The set difference of these two cuts is called the gap around $a$.

Let $a \in M(0) \backslash \operatorname{Hull}^{M}(\emptyset)$. We associate with $a$ two cuts of $M$,

$$
I_{a}^{+}=\inf \{u \in M: u>a \text { and } u \text { is definable }\}
$$

and

$$
I_{a}^{-}=\sup \{u \in M: u<a \text { and } u \text { is definable }\} .
$$

The set difference of these, i.e.,

$$
\Omega_{a}=I_{a}^{+} \backslash I_{a}^{-}
$$

is called the interstice around $a$. This notion was already isolated in [8]. Usually we work with a fixed interstice, hence we omit the subscript $a$. Obviously, if $M$ is recursively saturated and has nonstandard definable elements, then it has infinitely many interstices. In order to see this we pick a definable nonstandard $\alpha \in M$ and for given $n \in \mathbb{N}$ realize the type $\Gamma_{n}(x)$ expressing that $x$ is undefinable and $n \cdot \alpha<x<(n+1) \cdot \alpha$.

We let

$$
I_{0}(M)=\inf \{u \in M: u \text { is nonstandard and definable }\} .
$$

LEMMA 1.1. If $M$ is arithmetically saturated and has nonstandard definable elements then $I_{0}(M)>\mathbb{N}$.

The above lemma implies that every arithmetically saturated $M$ has the smallest interstice $\Omega_{\text {smallest }}=I_{0}(M) \backslash \mathbb{N}$. Also, every recursively saturated $M$ has the improper interstice, i.e., $M \backslash M(0)$.

Below we work with nondecreasing functions $F$ (in the most usual sense of the word, i.e., $\forall x, y[x \leq y \Rightarrow F(x) \leq F(y)]$ ). (In [1] we used, somewhat ambiguously, the name "increasing".)

Lemma 1.2. Let $\Omega=I^{+} \backslash I^{-}$be an interstice in $M$ and let $F$ be a nondecreasing function definable without parameters such that $I^{-}$is contained in the domain of $F$. Then $I^{-}$is closed under $F$ iff $I^{+}$is.

LEMmA 1.3. Let $M$ be recursively saturated and let $\Omega$ be an interstice. Then there exist two sequences $\alpha, \beta$ coded in $M$ such that

(i) $I^{-}=\sup \left\{\alpha_{n}: n \in \mathbb{N}\right\}$,

(ii) $I^{+}=\inf \left\{\beta_{n}: n \in \mathbb{N}\right\}$,

(iii) $\alpha_{n}$ is definable for every $n \in \mathbb{N}$,

(iv) $\beta_{n}$ is definable for every $n \in \mathbb{N}$.

Let $\Omega=I^{+} \backslash I^{-}$be an interstice in $M$. We let $\mathfrak{F}$ (or $\mathfrak{F}_{\Omega}$ if necessary) be the set of all nondecreasing functions which are definable without parameters and under which $I^{+}$is closed. 
The following lemma allows us to use $\mathfrak{F}$ in types provided $M$ is arithmetically saturated; in particular, Lemma 1.5 is a consequence of it. It is also the reason for which we sometimes work with arithmetically saturated models rather than with arbitrary recursively saturated ones.

LEMMA 1.4. If $M$ is arithmetically saturated and $\Omega=I^{+} \backslash I^{-}$is an interstice then the family $\mathfrak{F}$ is coded in $M$ (as the set of Gödel numbers).

Proof. Let $\alpha, \beta$ be as in Lemma 1.3. Let $S$ be a (partial inductive) satisfaction class for $M$. We let $j(F)=\min \left\{n: S\left(F\left(\alpha_{n}\right) \geq \beta_{n}\right)\right\}$ and $j(F)=0$ if $F$ is not a Gödel number of a term which is nondecreasing in the sense given by $S$. For this function $j$ we pick a nonstandard $u$ such that $j(F)>\mathbb{N}$ iff $j(F)>u$, using arithmetical saturation. Then $F \in \mathfrak{F}$ iff $j(F)>u$, so $\mathfrak{F}$ is coded in $M$.

Now we are ready to define intersticial gaps in $M(0)$. Let $\Omega=I^{+} \backslash I^{-}$ be an interstice in $M$. Let $u \in \Omega$. Exactly as we have done for $a>M(0)$, we put $\Omega(u)=\sup \{F(u): F \in \mathfrak{F}\}$ and $\Omega[u]=\sup \{w: \Omega(w)<u\}$. The set difference of these, i.e.,

$$
\operatorname{gap}^{\Omega}(u)=\Omega(u) \backslash \Omega[u]
$$

is called the intersticial gap around $u$. As usual we omit the superscript $\Omega$ unless it is really necessary. We also write "gap" rather than "intersticial gap" because the meaning will be determined by $a$, i.e., if $a>M(0)$ then we work with the usual gaps and if $a \in M(0)$ then we deal with intersticial gaps.

The following observation together with Lemma 1.4 shows that in the case of an arithmetically saturated model $M$, intersticial gaps are strictly smaller than interstices themselves.

Lemma 1.5. Let $M$ be recursively saturated and let $\Omega=I^{+} \backslash I^{-}$be an interstice in $M$. Then $\Omega$ itself is not an intersticial gap iff $\mathfrak{F}$ is in $\operatorname{SSy}(M)$.

Proof. Assume that $\Omega \neq \operatorname{gap}^{\Omega}(u)$ and let $w \in \Omega \backslash \operatorname{gap}^{\Omega}(u)$. We may assume that $u<w$. Then for nondecreasing $F$, we have $F \in \mathfrak{F}$ iff $F(u)<w$, so $\mathfrak{F}$ is coded in $M$. For the converse assume that $\mathfrak{F}$ is coded in $M$. Let $u \in \Omega$ and let $\beta$ be as in Lemma 1.3. Consider the type

$$
\Gamma(x)=\left\{x<\beta_{n}: n \in \mathbb{N}\right\} \cup\{F(u)<x: F \in \mathfrak{F}\} .
$$

This type is obviously consistent and in $\operatorname{SSy}(M)$, hence, it is realized by recursive saturation of $M$. Also, any of its realizations gives the second $\Omega$-gap.

We remark that if the interstice $\Omega$ is not an intersticial gap, then the set of intersticial gaps it contains is a dense linear order without endpoints. 
COROLLARY 1.6. If $M$ is an arithmetically saturated model of PA, then no interstice of $M$ is an intersticial gap.

Later we prove the converse of Corollary 1.6, i.e., if $M$ is recursively saturated and satisfies the condition of Corollary 1.6 then it is arithmetically saturated (see Theorem 5.1).

2. A combinatorial result. The goal of the next two sections is to work out a family of interstices in which selective types are realized, regular interstices. In this section we derive a combinatorial result needed for the construction. The idea is taken from Ketonen-Solovay [9]; indeed, from a certain point of view we push up one of their results ([9], Theorem 4.7) from $\omega^{2}$ to $\omega^{\omega}$ (and, in fact, to $\varepsilon_{0}$ ). We would like to point out that when using the particular version of the hierarchy, the so-called Hardy hierarchy, we are highly influenced by Zygmunt Ratajczyk's work (see [19], [16], [17] and [20]). Moreover, we refer to [2] for the main combinatorial lemma.

We shall work with the following version of the so-called Grzegorczyk hierarchy of quickly growing functions (see [5]). Let $G$ be a function which is either defined on the whole $\mathbb{N}$ or on some finite subset of $\mathbb{N}$. Assume moreover that $\forall x \in \operatorname{Dom}(G) x<G(x)$. As an example, let $A$ be a subset of $\mathbb{N}$ and let $G^{A}$ be the successor in the sense of $A$, i.e., the function with domain $A \backslash\{\max A\}$ which associates with every $a \in \operatorname{Dom}(G)$ the next element of $A$. We define a sequence $G_{n}$ of functions by putting $G_{0}(x) \simeq G(x)$ and $G_{n+1}(x) \simeq G_{n}^{x}(x)$, the $x$ th iteration of $G_{n}$, applied to $x$. Here iterations are defined in the usual manner, i.e., $G^{0}(x)=x$ and $G^{n+1}(x)=G^{n}(G(x))$. As usual, $\simeq$ means that both sides are defined and equal or both sides are undefined. But we shall use directly the equality sign. Observe that Ketonen and Solovay use a slightly different definition, i.e., $G_{n+1}(x)=G_{n}^{x+1}(x)$. For our purpose the definition as stated is more convenient.

Let $A$ be a finite subset of $\mathbb{N}$. Let $G^{A}$ denote the successor in the sense of $A$ and let $\left(G^{A}\right)_{n}$ denote Grzegorczyk's iterations of $G^{A}$. We say that $A$ is $n$-big iff $\left(G^{A}\right)_{n}(\min A)$ is defined. One may restate this definition in the following manner. A set $A$ is 0 -big iff it has at least two elements; $A$ is $(n+1)$-big iff there exists an increasing sequence $b_{0}, \ldots, b_{a}$ of elements of $A$ with $a=\min A, b_{0}=a$, and each interval $A \cap\left[b_{j}, b_{j+1}\right] n$-big.

The goal of this section is the following result.

Theorem 2.1. Let $A$ be an $(n+1)$-big set and let $A=\bigcup_{i<\min A} B_{i}$ be a partition of $A$ into at most $\min A$ parts. Then at least one of these parts is $n$-big.

As a matter of fact we shall work with the so-called Hardy hierarchy of quickly growing functions. Let $h$ be a function which satisfies the same 
condition as above, i.e., $\forall x \in \operatorname{Dom}(h) h(x)>x$. For every $\alpha<\varepsilon_{0}$ we define a function $h_{\alpha}$ by induction on $\alpha$. We put $h_{0}(x)=x$ and $h_{\alpha+1}(x)=h_{\alpha}(h(x))$.

Before defining the limit step we need to define, for each limit $\lambda<\varepsilon_{0}$, a sequence $\{\lambda\}(n)$ of ordinals convergent to $\lambda$ from below. We put $\{\omega\}(n)=n$, and, more generally, $\left\{\omega^{\alpha+1}\right\}(n)=\omega^{\alpha} \cdot n$. For limit $\gamma$ we put $\left\{\omega^{\gamma}\right\}(n)=$ $\omega^{\{\gamma\}(n)}$. Finally,

$\left\{\omega^{\alpha_{0}} \cdot m_{0}+\ldots+\omega^{\alpha_{s}} \cdot m_{s}\right\}(n)=\omega^{\alpha_{0}} \cdot m_{0}+\ldots+\omega^{\alpha_{s}} \cdot\left(m_{s}-1\right)+\left\{\omega^{\alpha_{s}}\right\}(n)$,

where $\lambda=\omega^{\alpha_{0}} \cdot m_{0}+\ldots+\omega^{\alpha_{s}} \cdot m_{s}$ is the Cantor normal form expansion of $\lambda$, i.e., $\alpha_{0}>\ldots>\alpha_{s}$. It is easy to see that these conditions determine exactly one sequence $\{\lambda\}(n): n$, for each $\lambda<\varepsilon_{0}$. Observe also that Ketonen and Solovay [9] use a slightly different notion of $\{\lambda\}(n)$. We shall call the sequence $\{\lambda\}(n)$ the fundamental sequence for $\lambda$. It is possible to extend fundamental sequences to larger ordinals (cf. e.g. [18]). For example let $\omega_{0}=\omega$ and $\omega_{n+1}=\omega^{\omega_{n}}$. Then $\left\{\varepsilon_{0}\right\}(n)=\omega_{n}$ is a fundamental sequence for $\varepsilon_{0}$.

Now we are ready to define $h_{\lambda}$ for $\lambda$ limit. We simply put $h_{\lambda}(x)=$ $h_{\{\lambda\}(x)}(x)$. This completes the definition of Hardy hierarchy based on $h$. Of course, we also extend Grzegorczyk's hierarchy to the so-called Grzegorczyk-Wainer hierarchy by putting $G_{0}(x)=G(x), G_{\alpha+1}(x)=G_{\alpha}^{x}(x)$ and $G_{\lambda}(x)=G_{\{\lambda\}(x)}(x)$ for $\lambda$ limit.

This notion allows us to define a set $A$ of natural numbers to be $\alpha$-large in the same manner as above. That is, $A$ is $\alpha$-large iff $\left(h^{A}\right)_{\alpha}(a)$ is defined, where $h^{A}$ denotes the successor in the sense of $A$ and $a=\min A$. One can restate this definition of largeness in the following manner. A set $A$ is 0-large iff it is nonempty. $A$ is $(\alpha+1)$-large iff $A \backslash\{\min A\}$ is $\alpha$-large. $A$ is $\lambda$-large, $\lambda$ limit, iff it is $\{\lambda\}(\min A)$-large. Observe that Ketonen and Solovay [9] use a slightly different notion of largeness. Indeed, they define their notion by reverse induction on the natural enumeration (i.e., in the increasing order) of $A$. But the difference between these two notions is only technical.

Lemma 2.2. Let $h$ be as above. Then for every $\alpha$ and every $\beta \gg \alpha$, $h_{\beta+\alpha}=h_{\beta} \circ h_{\alpha}$.

Proof. By induction on $\alpha$.

LEMMA 2.3. Let $h$ be as above and denote by $h_{\alpha}$ the $\alpha$ th iterate of $h$ in the Hardy sense and by $G_{\alpha}$ its $\alpha$ th iterate in the Grzegorczyk sense. Then $G_{\alpha}=h_{\omega^{\alpha}}$.

Pr o of. Immediate by induction on $\alpha$, using Lemma 2.2.

In other words, a set $A$ is $\alpha$-big iff it is $\omega^{\alpha}$-large. Granted this connection we see that in order to prove Theorem 2.1 it suffices to show the following result and apply it below $\omega^{\omega}$. 
Theorem 2.4. If $A$ is $\omega^{\alpha+1}$-large and $A=\bigcup_{i<\min A} B_{i}$ is a partition of $A$ into at most $a=\min A$ parts then at least one $B_{i}$ is $\omega^{\alpha}$-large.

We write "into at most $\min A$ parts" because we allow some $B_{i}$ to be empty. We refer the reader to the paper [2] for a proof of Theorem 2.4.

Of course, Theorem 2.1 follows from Theorem 2.4, by Lemma 2.3.

We remark that all these results are provable in PA. More exactly, first, they are expressible in PA. Indeed, definitions by transfinite induction (like that of Hardy hierarchy) are expressible in PA using notation systems for ordinals and the recursion theorem (cf. [21] for the recursion-theoretic approach and [6] or [18] for the proof-theoretic approach to this sort of problems). Moreover, if we fix $n \in \mathbb{N}$, then all the above results are provable for ordinals less than $\omega_{n}$ because PA proves transfinite induction over $\omega_{n}$, by the classical result due to G. Gentzen. See [6], p. 138, Theorem 5.2 in [16] or $[23]$ for more in this direction.

3. Regular interstices. The goal of this section is to describe a class of interstices (regular interstices) in which selective types are realized. Let $\Omega=I^{+} \backslash I^{-}$be an interstice in a countable arithmetically saturated model of PA. Let $\mathfrak{F}$ denote, as usual, the set of all nondecreasing functions, definable without parameters, under which $I^{-}$is closed.

Definition 3.1. An $M$-finite set $A$ is $\Omega$-large if it is definable without parameters and $A \cap \Omega \neq \emptyset$.

We remark that if $A$ is definable without parameters then $A$ is $\Omega$-large iff $I^{-}$is closed under the successor in the sense of $A$, i.e., for every $a \in A$ if $a<I^{-}$then the next element of $A$ is also smaller than $I^{-}$. Indeed, otherwise for some definable $\alpha<I^{-}$we would have $\forall a \in A a \leq \alpha \vee a>I^{-}$. But then "the smallest element of $A$ which exceeds $\alpha$ " is definable and in $\Omega$, which is impossible.

Definition 3.2. An interstice $\Omega=I^{+} \backslash I^{-}$is regular iff for every $\Omega$-large $A \in M$ and every partition $A=\bigcup_{i<\alpha} A_{i}$ definable without parameters with $\alpha<I^{-}$, at least one part $A_{i}$ is $\Omega$-large.

It is easy to see that the smallest interstice, $\Omega_{\text {smallest }}=I_{0}(M) \backslash \mathbb{N}$, where $I_{0}(M)$ denotes the greatest lower bound of all nonstandard definable elements of $M$, is regular. Let us recall that for many models $M$ of PA, $I_{0}(M)>\mathbb{N}$, so this interstice exists. By Lemma 1.1, this happens always if $M$ is arithmetically saturated. See [8] and [1] for more about $\Omega_{\text {smallest }}$. The construction of this section gives many more examples of regular interstices.

THEOREM 3.3. Let $M$ be a countable recursively saturated model of some false completion of PA and let $A \in M$ be a definable set which is n-big (in 
the sense introduced in §2) for some nonstandard $n \in M$. Then there exists a regular interstice $\Omega=I^{+} \backslash I^{-}$such that $A \cap \Omega \neq \emptyset$.

Proof. Let $A, n \in M$ satisfy the assumption. We enumerate as $P_{j}$ : $j \in \mathbb{N}$ all definable partitions of $A$ in $M$ in such a way that each definable partition occurs in this enumeration infinitely often. To be more specific, we use a (partial inductive) satisfaction class and an enumeration of formulas to encode this enumeration inside $M$. Observe that $\max \{n: A$ is $n$-big $\}$ is definable because $A$ is definable.

Pick the first of these partitions, say $P_{i_{0}}: A \rightarrow b_{i_{0}}$, which satisfies $b_{i_{0}} \leq \min A$. By Theorem 2.1 there exists $A_{0}$ which is $(n-1)$-big and homogeneous for $P_{i_{0}}$. We continue in the same fashion, that is, we consider the first (not used up to now) partition $P_{i_{1}}$ which satisfies $P_{i_{1}}: A_{0} \rightarrow b_{i_{1}}$ with $b_{i_{1}} \leq \min A_{0}$ and take a homogeneous set $A_{1}$, and so on. Observe that this procedure is performed in $(M, S)$, where $S$ is a partial inductive satisfaction class for $M$ (from a standard definition of the partition we obtain a standard definition of a homogeneous set), hence, the sequence $A_{j}$ is coded in $M$. We put $I^{-}=\sup \left\{\min A_{i}: i \in \mathbb{N}\right\}$ and see that this cut determines an interstice $\Omega$ with $I^{+}=\inf \left\{e: e\right.$ is definable and greater than $\left.I^{-}\right\}$. We claim that this interstice is regular.

Define $d_{r}=\min A_{r}$ and $e_{r}=\max A_{r}$. Obviously, both sequences are coded, the first is nondecreasing and the second is nonincreasing. Moreover, $d_{r}<d_{r+1}$ for infinitely many $r$. The reason is that, given $r$, the partition

$$
A=\left(A \backslash A_{r}\right) \cup\left\{\min A_{r}\right\} \cup\left(A_{r} \backslash\left\{\min A_{r}\right\}\right)
$$

occurs in the enumeration infinitely often, so had to be considered in the $k$ th step for some $k>r$. It follows that $d_{r}<d_{k}$, hence $d_{s}<d_{s+1}$ for some $s$ with $r \leq s<k$. The same reasoning shows that $e_{r}>e_{r+1}$ for infinitely many $r$.

We have put $I^{-}=\sup \left\{d_{r}: r \in \mathbb{N}\right\}$ above. We claim that $I^{+}=\inf \left\{e_{r}\right.$ : $r \in \mathbb{N}\}$. To see this suppose that some definable $u$ is between $I^{-}$and $\inf \left\{e_{r}\right.$ : $r \in \mathbb{N}\}$, consider the partition $A=\{x \in A: x \leq u\} \cup\{x \in A: u<x\}$ and see that either the homogeneous set is below or above $u$, so it is not between the two cuts we are talking about.

Let $B$ be an $\Omega$-large set and let $B=\bigcup_{i<u} B_{i}$ be a partition of $B$ definable without parameters with $u<I^{-}$. First we thin down $B$ to some smaller set $C$. We write $A=\left\{w_{0}, \ldots, w_{e-1}\right\}$ in increasing order. We put $j_{0}=0$ and $c_{0}=\min \left\{b \in B: w_{j_{0}} \leq b\right\}$. We continue in the same fashion, i.e., we set $j_{i+1}=\min \left\{j: b_{j_{i}}<w_{j}\right\}$ and $c_{i+1}=\min \left\{b \in B: w_{j_{i+1}} \leq b\right\}$. This induction breaks off after, say, $s$ steps (either there is no $j_{s}$ or there is no $b_{j_{s}}$ ). We let $C=\left\{c_{i}: i<s\right\}$.

We claim that $C$ is $\Omega$-large. Indeed, $I^{-}<w_{t}<I^{+}$for some $t$ as $A$ is $\Omega$ large. Also, $B$, being $\Omega$-large, is cofinal in $\Omega$, so there exists $b \in B \cap \Omega$ which 
is greater than $w_{t}$. The same reasoning shows that the set $D=\left\{w_{j_{i}}: i<s\right\}$ is $\Omega$-large.

The partition $B=\bigcup_{i<u} B_{i}$ induces a partition $C=\bigcup_{i<u}\left(C \cap B_{i}\right)$. This partition induces one more partition of $D$. We put $w_{j_{i}} \in D_{m}$ iff $c_{i} \in C \cap B_{m}$. Thus, we have got a partition $D=\bigcup_{m<u} D_{m}$ and by construction, one of $D_{m}$ 's is $\Omega$-large. But then, for the same $m, C \cap B_{m}$ is $\Omega$-large, and so $B_{m}$ is $\Omega$-large as well.

The following is the main result about regular interstices.

THEOREM 3.4. Let $M$ be a countable recursively saturated model of PA and let $\Omega=I^{+} \backslash I^{-}$be a regular interstice in $M$. Then the type of some $b \in \Omega$ is selective.

P r o of. First we construct a selective type $q(\cdot)$. We proceed by induction, in each step we decide what to do with a formula and with a term. The inductive condition is as follows:

$$
\varphi \in q \Rightarrow \exists x<I^{-} \varphi(x) \& \forall x<I^{-}\left[\varphi(x) \Rightarrow \exists y>x\left(\varphi(y) \& x<y<I^{-}\right)\right] .
$$

In other words, $I^{-}$is closed under the successor in the sense of $\{x: \varphi(x)\}$, i.e., this set is $\Omega$-large.

It is easy to check that if $\varphi$ has this property then for every formula $\psi$, at least one of $\varphi \& \psi, \varphi \& \neg \psi$ has this property, so let us turn to terms. Assume that $\varphi$ has the above property and let a term $t(\cdot)$ be given. We define inside $M$ a sequence $x_{i}$ by the following induction. We put

$$
x_{0}=\min x: \varphi(x) \quad \text { and } \quad x_{j+1}=\min x: \varphi(x) \& \forall i \leq j t(x) \neq t\left(x_{i}\right) .
$$

We consider two cases.

CASE 1: $\forall i x_{i}<I^{-} \Rightarrow x_{i+1}<I^{-}$. Then we put into $q$ the formula $\exists i x=x_{i}$ and see that this formula still has the above property.

CASE 2: $\exists i x_{i}<I^{-} \& x_{i+1}>I^{-}$. We pick a definable $\alpha$ with $x_{i}<\alpha<I^{-}$ and see that $x_{i}$ is definable and hence so is $x_{i+1}$. It follows that $x_{i+1}>I^{+}$. Let $B=\left\{x \in\left[x_{i}, x_{i+1}\right]: \varphi(x)\right\}$. By this remark, $B$ is $\Omega$-large. Moreover,

$$
\forall x \in B \exists e<x_{i} t(x)=t(e) \& \varphi(e) .
$$

It follows that we have a partition

$$
B=\bigcup_{e<x_{i}}\{x \in B: t(x)=t(e)\} .
$$

By regularity of $\Omega$, there exists $e<x_{i}$ such that $\{x \in B: t(x)=t(e)\}$ is $\Omega$ large. In particular, $e$ is definable. We put into $q$ the formula $\varphi(x) \& t(x)=e$.

Granted these remarks there is no problem in constructing the type $q$. Obviously, it is selective. Moreover, it is easy to check that it is realized by some $b \in \Omega$. 
Recall from [1] that an interstice $\Omega$ is very good if for every $F \in \mathfrak{F}$ the function $x \mapsto F^{x}(x)$ is in $\mathfrak{F}$ as well.

Proposition 3.5. An interstice $\Omega=I^{+} \backslash I^{-}$is very good iff for every $\Omega$-large set $A$, the cardinality of $A$ is $>I^{+}$.

Proof. Let $\Omega$ be very good and let $A \cap \Omega \neq \emptyset$. Assume that $\operatorname{card}(A)<$ $I^{-}$. Let $h=h^{A}$ be the successor in the sense of $A$. Then $h \in \mathfrak{F}$ because $A$ is $\Omega$-large. Let $a=\min A$. Iterate $h \operatorname{card}(A)$ times to obtain something greater than $I^{+}$, contrary to $\Omega$ being very good.

For the converse assume that $A \cap \Omega \neq \emptyset \Rightarrow \operatorname{card}(A)>I^{+}$but $\Omega$ is not very good. Pick $F \in \mathfrak{F}$ such that the function $x \mapsto F^{x}(x)$ is not in $\mathfrak{F}$. Then for some definable $\alpha<I^{-}, F^{\alpha}(\alpha)=\beta>I^{+}$. Let $A=\left\{\alpha, F(\alpha), \ldots, F^{\alpha}(\alpha)\right\}$. This set has cardinality $\alpha+1<I^{-}$, so it cannot intersect $\Omega$. Hence for some $j, F^{j}(\alpha)<I^{-}<I^{+}<F^{j+1}(\alpha)$, contrary to $F \in \mathfrak{F}$.

COROLLARY 3.6. Every regular interstice is very good.

A similar argument also establishes

Lemma 3.7. Let $\Omega$ be a regular interstice and let $A$ be an $\Omega$-large set. Then the function which enumerates $A$ in increasing order is in $\mathfrak{F}_{\Omega}$.

Theorem 3.4 admits the following weak converse.

THEOREM 3.8. If $\Omega$ is a very good interstice which contains an element a such that $\operatorname{tp}(a)$ is selective then $\Omega$ is regular.

Proof. Let $a \in \Omega$ realize a selective type. Assume that $\Omega$ is not regular. Let $B$ be an $\Omega$-large set and let a definable partition $B=\bigcup_{i<\alpha} B_{i}$ be given with $\alpha<I^{-}$and such that no $B_{i}$ is $\Omega$-large. Pick $i<\alpha$ such that $\min \{x \in B: x \geq a\}$ is in $B_{i}$. If $i$ is definable then $B_{i}$ is $\Omega$-large as it intersects $\Omega$. Hence $i$ is not definable. But $i=t(a)$ for some term $t(\cdot)$ and by selectivity of $\operatorname{tp}(a), a=s(i)$ for some term $s(\cdot)$. It follows that $a$ is in the set $\{x: \exists y y<\alpha \& x=s(y)\}$, so this set is $\Omega$-large, which is impossible by Proposition 3.5: indeed, its cardinality is at most $\alpha$.

Let us remark that Theorem 3.4 does not admit a full converse. There are many interstices in $M$ which are not regular but realize some selective types. We give an example below, but point out that the situation is slightly unclear. That is, we do not know the exact description of interstices which realize selective types.

THEOREM 3.9. Every recursively saturated model of some false completion of $\mathrm{PA}$ has a nonregular interstice in which some selective type is realized.

Proof. Let $A \in M$ be any set definable without parameters of nonstandard cardinality. Let $B=\{a+\operatorname{card}(A): a \in A\}$. Then $B$ is not 2-big, so no 
interstice $\Omega$ such that $B$ is $\Omega$-large is regular. But the standard construction involving the finite version of the Ramsey theorem gives a 2-indiscernible (hence, selective) type $q(\cdot)$ such that the formula $v \in B$ is in $q$. Let $b$ realize such a type $q$. Then the interstice of $b$ satisfies our demand.

Our final goal in this section is to show that the converse to Lemma 3.6 is false; indeed, we show below that there exist very good interstices with no element realizing any selective type.

Lemma 3.10. Let $\Omega=I^{+} \backslash I^{-}$be an interstice in a countable arithmetically saturated model of some false completion of PA. Then the following are equivalent:

(i) for every $\Omega$-large $A, \operatorname{card}(A)>I^{+}$,

(ii) every $\Omega$-large $A$ is $\omega$-large in the Hardy sense.

Proof. One direction is obvious: indeed, if $A$ is $\Omega$-large and $\operatorname{card}(A)>$ $I^{+}$then $\min A<I^{-}<I^{+}<\operatorname{card}(A)$, so $A$ is $\omega$-large. For the converse assume that some $A$ is $\Omega$-large, but $\operatorname{card}(A)<I^{+}$. Then $\operatorname{card}(A)<I^{-}$: indeed, $A$ is definable without parameters, hence its minimum is as well. Let $B=\{x \in A: \operatorname{card}(A)<x\}$. Then $B$ is $\Omega$-large: indeed, each element of $A \backslash B$ is smaller than $\operatorname{card}(A)<I^{-}$, so $B \cap \Omega \neq \emptyset$. On the other hand, $\operatorname{card}(B) \leq \operatorname{card}(A)<\min B$, so $B$ is not $\omega$-large.

TheOREM 3.11. Let $M \not \models \operatorname{Th}(\mathbb{N})$ be a recursively saturated model of PA. Then there is a very good interstice $\Omega$ which does not contain any element realizing a selective type.

COROLlARY 3.12. Every recursively saturated model of some false completion of PA has an interstice which is very good but not regular.

Proof. Immediate by Theorems 3.11 and 3.4.

Before proving Theorem 3.11 we need another combinatorial notion. We define (in PA) the notion of an $m$-gentried function $f:[a, b) \rightarrow D$, where $D$ is a (finite) set. Roughly speaking, $f$ is gentried if it has a large domain.

First, we say that $f:[a, b) \rightarrow D$ is 0 -gentried if $[a, b)$ is $\omega$-large. Inductively, $f:[a, b) \rightarrow D$ is $(m+1)$-gentried if, whenever $X \subseteq[a, b)$ and either

(i) $f$ is constant or one-one on $X$, or

(ii) $X$ is not $\omega$-large,

then there is $[c, d) \subseteq[a, b)$ such that $[c, d) \cap X=\emptyset$ and $f \uparrow[c, d)$ is $m$-gentried. It is obvious that, for any $m$, if $f$ is $m$-gentried, then the domain of $f$ is $\omega$-large.

Lemma 3.13 (in PA). For every $z$ and $x$, there are $y$ and $a$-gentried function $f:[x, y) \rightarrow[0, z]$. 
Proof. The proof is by induction on $z$. If $z=0$, then let $y=2 x+1$, and thus $[x, y)$ is $\omega$-large, and let $f$ be the function which is constantly 0 on $[x, y)$.

Assume we have proved the lemma for some $z$; we will prove it for $z+1$. We define a sequence $x_{0}<x_{1}<x_{2}<\ldots$ and a sequence of functions $f_{i}$ : $\left[x_{i}, x_{i+1}\right) \rightarrow[0, z+1]$ as follows. Let $x_{0}=x$. Having $x_{i}$, we let $x_{i+1}>x_{i}$ and $f_{i}:\left[x_{i}, x_{i+1}\right) \rightarrow[0, z+1]$ be such that $f_{i}$ is $z$-gentried and $f_{i}$ " $\left(\left[x_{i}, x_{i+1}\right)\right) \subseteq$ $[0, z+1] \backslash\{r\}$, where $r$ is the remainder when $i$ is divided by $z+2$. Let $t$ be such that $\left\{x_{1}, \ldots, x_{t}\right\}$ is $\omega$-large and $t>z+2$. Then let $y=x_{t}$ and let $f=\bigcup_{i<t} f_{i}$.

We claim that $f:[x, y) \rightarrow[0, z+1]$ is $(z+1)$-gentried. Let $X \subseteq[x, y)$. We have three cases to consider. In each of these cases, we will find an $i<t$.

First, suppose that $f$ is constant on $X$. Let $i \in[0, z+1]$ be the constant value of $f$. Second, suppose that $f$ is one-one on $X$. Then $\operatorname{card}(X) \leq z+2$, so there is some $i \leq z+2$ such that $\left[x_{i}, x_{i+1}\right) \cap X=\emptyset$.

Finally, suppose that $X$ is not $\omega$-large. Let $A=\{i+1 \leq t: X \cap$ $\left.\left[x_{i}, x_{i+1}\right) \neq \emptyset\right\}$, so $A$ is not $\omega$-large. Thus, there is $i<t$ such that $X \cap$ $\left[x_{i}, x_{i+1}\right) \neq \emptyset$.

In each case, let $[c, d)=\left[x_{i}, x_{i+1}\right)$, so that $f\left\lceil[c, d)\right.$ is $f_{i}$. Clearly, $[c, d) \cap$ $X=\emptyset$ and $f_{i}$ is $m$-gentried.

Proof of Theorem 3.11. Let $D_{0}, D_{1}, D_{2}, \ldots$ be a recursive list of all 0 definable subsets of $M$. Let $d$ be a nonstandard definable element of $M$, and let $f:\left[a_{0}, b_{0}\right) \rightarrow[0, d]$ be the first (in some canonical order) definable $d$ gentried function. By the lemma, there is such an $f$; clearly, it is 0-definable. Using the lemma repeatedly, we find $\left[a_{0}, b_{0}\right) \supseteq\left[a_{1}, b_{1}\right) \supseteq\left[a_{2}, b_{2}\right) \supseteq \ldots$ such that, for each $i \in \mathbb{N}, f\left\lceil\left[a_{i}, b_{i}\right)\right.$ is $(d-i)$-gentried, and if either $f$ is constant or one-one on $D_{i}$ or $D_{i}$ is not $\omega$-large, then $\left[a_{i+1}, b_{i+1}\right) \cap D_{i}=\emptyset$. Moreover, by always taking the first pair $\left[a_{i}, b_{i}\right)$ which works we find that each $a_{i}$ and $b_{i}$ is definable and that the two sequences $\left\langle a_{i}: i \in \mathbb{N}\right\rangle$ and $\left\langle b_{i}: i \in \mathbb{N}\right\rangle$ are recursive.

Let $\Omega=\left\{x \in M: a_{i}<x<b_{i}\right\}$. Then $\Omega \neq \emptyset$ by the recursive saturation of $M$. It is easy to see that $\Omega$ is an interstice. For, if any $c \in M$ is definable, then for some $i, D_{i}=\{c\}$, which is not $\omega$-large, so that $c \notin \Omega$. Clearly, $\Omega$ is very good, by Lemma 3.10. Finally, we show that if $c$ realizes a selective type, then $c \notin \Omega$. There is some $i \in \mathbb{N}$ such that $c \in D_{i}$ and $f$ is either constant or one-one on $D_{i}$. But then $\left[a_{i+1}, b_{i+1}\right) \cap D_{i}=\emptyset$, so that $c \notin \Omega$.

4. Selective types and maximality of basic subgroups. The main (from the point of view of automorphisms) property of selective types is Theorem 3.4 of [10] which states that if $a>M(0)$ then $\operatorname{tp}(a)$ is selective iff the stabilizer $G_{a}$ is a maximal subgroup of $G=\operatorname{Aut}(M)$. Unfortunately, 
the proof given in [10] does not work for $a<M(0)$. The exact description of those $a \in M(0)$ with maximal stabilizer is not known. But observe that there exist $a \in M(0)$ such that $G_{a}$ is maximal. The reason is that the proof of Theorem 2.1 of [10] (stating that $G_{a}$ is strongly maximal $\operatorname{iff} \operatorname{tp}(a)$ is 2 -indiscernible) works without the assumption that $a>M(0)$. Also, there is no problem in finding $a \in M(0)$ realizing 2-indiscernible types: indeed, the standard construction involving the (finite version of) Ramsey theorem yields such types, and hence the appropriate elements. But we point out that it is not clear in which interstices such elements exist.

The following construction yields a selective type which does not determine a maximal subgroup.

The argument depends on the so-called canonical Ramsey theorem of Erdös and Rado [3]. Let us state it. If $S$ is any set, we denote by $[S]^{r}$ the set of all $r$-element subsets of $S$. If $S$ is linearly ordered then we may identify $[S]^{r}$ with the set of all increasing $r$-tuples of elements of $S$. A function (coloring) $\chi$ with domain $[S]^{r}$ is canonical if for some $V \subseteq\{1, \ldots, r\}$, we have $\chi\left(\left\langle a_{1}, \ldots, a_{r}\right\rangle\right)=\chi\left(\left\langle b_{1}, \ldots, b_{r}\right\rangle\right)$ iff $a_{i}=b_{i}$ for all $i \in V$.

THEOREM 4.1. For every $k, r$ there exists $n$ such that for every coloring of $[n]^{r}$ there exists $T \subset\{0, \ldots, n-1\}$ of cardinality at least $k$ on which the coloring is canonical.

We stress that this result concerns colorings into arbitrarily many colors. The result as stated is taken from [4], p. 133. But it is proved there only for $r=2$; for the complete treatment we refer to [3].

Below we shall work with partitions of the usual Cartesian products $\times_{i<m} X_{i}$ of (finite) sets, rather than sets of the form $[X]^{m}$; our first task is to derive the appropriate partition result for this case from Theorem 4.1.

Just as with $[S]^{r}$ we say that a function $f$ with domain $\times_{i<m} X_{i}$ is canonical iff there exists a subset $I \subseteq(<m)$ such that for all $a, b \in \times_{i<m} X_{i}$, $f(a)=f(b)$ iff $a_{i}=b_{i}$ for all $i \in I$. If one thinks of $\times_{i<m} X_{i}$ as of a parallelepiped then this means that for some subset $I$ of the coordinate set, $a, b$ have the same color iff $a, b$ have the same coordinates $i \in I$, i.e., they might differ only in coordinates $i \notin I$.

Lemma 4.2. For every $m, k$ there exists $n$ so that for every sequence $X_{1}, \ldots, X_{m}$ of sets of cardinality $\geq n$ each and every function $f$ with domain $\times_{i=1}^{m} X_{i}$ there exist sets $Z_{1}, \ldots, Z_{m}$ with each $Z_{i}$ being a subset of $X_{i}$ of cardinality at least $k$ such that $f$ is canonical on $Z=\times_{i=1}^{m} Z_{i}$.

P r o o f. Given $m, k$ we find $n$ which satisfies the condition of Theorem 4.1 for $k$ and $r=m k$. Let sets $X_{1}, \ldots, X_{m}$ be given. We may assume that they are all equal, say $B$, and $\operatorname{card}(B)=n$. Thus, $f$, being defined on $B^{m}$, is defined in particular on $[B]^{m}$. By Theorem 4.1, there exists a set $A \subseteq B$ such 
that $\operatorname{card}(A) \geq m k$ and $f$ is canonical on the set of all $m$-element subsets of $A$. We take $Z_{1}$ to be the set of first $k$ elements of $A, Z_{2}=$ the set of next $k$ elements of $A$, etc. Let $Z$ be the product of the $Z_{i}$. Clearly, $f$ is canonical on $Z$.

Lemma 4.3. Given $m, k$ there exists $n$ such that whenever $X_{i}$ for $i \in$ $\{0, \ldots, 2 m\}$ are sets of cardinality at least $n$ and $f$ is a function with domain $X=\times_{i \leq 2 m} X_{i}$, there exist $Y_{i} \subseteq X_{i}$ such that $m+1$ of $Y_{i}$ 's have cardinality at least $k$, others are singletons and $f$ is either constant or one-one on $Y=$ $\times_{i \leq 2 m} Y_{i}$.

Pr o o f. Given $m, k$ we pick $n$ which satisfies the condition of Lemma 4.2 for $2 m+1, k$. Let sets $X_{i}: i \leq 2 m$ be given, let $X=\times_{i=0}^{2 m} X_{i}$ and let $f$ be a function defined on $X$. By Lemma 4.2 there are $Z_{i} \subseteq X_{i}$, each of cardinality at least $k$, such that $f$ is canonical on $Z$, the product of $Z_{i}$ 's. Let $I$ be a subset of $\{0, \ldots, 2 m+1\}$ such that for all $a, b \in Z, f(a)=f(b)$ iff $a_{i}=b_{i}$ for all $i \in I$. Either $I$ or its complement is of power $>m$. If $\operatorname{card}(I)>m$ we let $Y_{i}=Z_{i}$ for $i \in I$ and let the rest of $Y_{i}$ be singletons. Otherwise we let $Y_{i}=Z_{i}$ for $i \notin I$ and the other $Y_{i}$ be singletons. In the first case $f$ is constant on $Y$ (the product of $Y_{i}$ 's) and in the second case it is one-one on $Y$.

Observe that all these combinatorial results are provable in PA. Granted them we construct a type. So let $M$ be a countable recursively saturated model of some false completion of PA. Pick nonstandard definable $m, n \in M$. We may assume that $m$ is odd, otherwise we take $m-1$. Let $t_{s}(\cdot)$ be a recursive enumeration of all parameter-free terms in one free variable as shown. Begin with the product $n^{m}$ (we identify $n$ with the set of elements $<n$ ). Begin with $t_{0}$. Let $k$ be as in Lemma 4.3. Obviously, $k$ is nonstandard. Choose a family $Y_{i}: i<m$ such that each $Y_{i}$ is a subset of $n,(m+1) / 2$ of them are of cardinality $\geq k$, others are singletons and $t_{0}$ is either one-one or constant on the product $\times_{i \leq m} Y_{i}$. Then this product is isomorphic to the product of the form $k^{(m+1) / 2}$, so Lemma 4.3 applies. We apply it to the next term and so on. This procedure determines the type $\Gamma(x)$ expressing that $x$ is in all of the products obtained in this manner.

Observe that the type $\Gamma(x)$ is complete. Indeed, if $\varphi(\cdot)$ is a formula then the term $[y=0 \& \varphi(x)] \vee[y=1 \& \neg \varphi(x)]$ is one of the terms $t_{s}$, so is constant on some product belonging to our type. Clearly, it cannot be one-one on a set of nonstandard cardinality. Also clearly $\Gamma$ is selective. Finally, it is obvious that $\Gamma$ is in the standard system of $M$ (it was constructed recursively from the complete diagram of $M$ and the enumeration of terms), so it is realized in $M$. 
Let $a \in M$ realize this type. We show that the stabilizer $G_{a}$ is not maximal. The reason is that in each step we passed to a smaller product, at about half coordinates we had singletons and at others we still had sets of nonstandard cardinality. It follows that there exists $b \neq a$ realizing $\Gamma$ and such that $a, b$ differ just in one coordinate. Also there exists $c$ realizing $\Gamma$ such that $a, c$ differ in a nonstandard number of coordinates. Let $f, g \in \operatorname{Aut}(M)$ be such that $g(a)=b$ and $f(a)=c$. Clearly, $f$ cannot be in the group generated by $G_{a}$ and $g$. Indeed, for $n \in \mathbb{Z}, g^{n}(a)$ differs from $a$ in a standard finite number of coordinates, and, hence, the same is true for each element of the subgroup of $G$ generated by $G_{a}$ and $g$. Summing up, we obtain the following result which refutes a conjecture from [10].

THEOREM 4.4. If $M$ is a countable recursively saturated model of some false completion of $\mathrm{PA}$ then there exists $a \in M(0)$ realizing a selective type but whose stabilizer is not maximal.

It is not clear whether there may be $a \in \Omega_{\text {smallest }}$ whose type is selective but $G_{a}$ is not maximal.

5. A remark on the non-arithmetically saturated case. In this section we prove the converse to Corollary 1.6. That is, we prove

THEOREM 5.1. If $M$ is a recursively saturated model of some false completion of PA which is not arithmetically saturated then $M$ has an interstice which is equal to its unique gap.

We begin with the following (well known) lemma.

Lemma 5.2. Let $M$ be a recursively saturated model of PA. Then $M$ is arithmetically saturated iff the standard system of $M, \operatorname{SSy}(M)$, is closed under arithmetical definability (i.e., definability in $\mathbb{N}$ ).

Pr o o f. See, e.g., [12].

COROLLARY 5.3. If $M$ is recursively saturated but not arithmetically saturated then there exists a set $A \subseteq \mathbb{N}$ which is not in $\operatorname{SSy}(M)$ but there exists an enumeration of $A$ which is in $\operatorname{SSy}(M)$.

We remark that the conclusion means that $A=\left\{r_{0}, r_{1}, \ldots\right\}$ for some coded set of pairs $\left\langle k, r_{k}\right\rangle$. Corollary 5.3 is well known; we leave its proof to the reader.

We begin the proof of Theorem 5.1. So let $M$ satisfy the assumption. Pick $A \notin \mathrm{SSy}(M)$ which has a coded enumeration, $A=\left\{r_{0}, r_{1}, \ldots\right\}$. Let $d_{0}, d_{1}, \ldots$ be a recursive list of all constant terms. Fix also a nonstandard 
definable element $c \in M$. We define a recursive sequence $\alpha_{0}, \alpha_{1}, \ldots$ of definable elements of $M$. Let $\alpha_{0}=0$. Let also

$$
\alpha_{k+1}= \begin{cases}\alpha_{k}+\left(2 r_{k}+2\right) c^{c-k} & \text { if } d_{k} \leq \alpha_{k}+\left(2 r_{k}+2\right) c^{c-k} \\ \alpha_{k}+\left(2 r_{k}+1\right) c^{c-k} & \text { otherwise }\end{cases}
$$

The sequence $\alpha_{k}$ is coded and increasing. We also let $\beta_{k}=\alpha_{k}+c^{c-k+1}$. We put $I^{-}=\sup \left\{\alpha_{k}: k \in \mathbb{N}\right\}$ and $I^{+}=\inf \left\{\beta_{k}: k \in \mathbb{N}\right\}$. These sequences determine an interstice $\Omega$. Indeed, by high-school algebra every definable element of the form $d_{k}$ is either smaller than $\alpha_{k+1}$ or greater than $\beta_{k+1}$, so it is not in $\Omega$. We assert that $\mathfrak{F}_{\Omega}$ is not coded in $M$, hence $\Omega$ is an intersticial gap by Lemma 1.5 .

It will be convenient to have one more notation. Let the sequence $s_{k}$ be such that

$$
\alpha_{k}=s_{0} c^{c}+s_{1} c^{c-1}+\ldots+s_{k} c^{c-k}
$$

each $s_{k}$ being either $2 r_{k}+1$ or $2 r_{k}+2$.

In order to check that $\Omega$ has the desired property we define a function $f$ on $\left[0, c^{c+1}\right] \times[0, c]$. We let $f(0, i)=0$ for all $i \leq c$. In order to define $f(x, i)$ for $x>0$ pick $x \in\left[1, c^{c+1}\right]$ and express it in base $c$ :

$$
x=b_{0}(x)+b_{1}(x) c+b_{2}(x) c^{2}+\ldots+b_{c}(x) c^{c},
$$

where each $b_{k}(x)<c$. Let $k=k(x)$ be the least such that $b_{k}(x) \neq 0$. Let $f(x, i)=x$ if $b_{k}(x)$ is neither $2 i+1$ nor $2 i+2$ and $f(x, i)=x+c^{c-k+1}$ otherwise. Let also $f_{r}(x)=f(x, r)$.

We claim that $I^{-}$is closed under $f_{r}$ iff $r \notin A$. Assume firstly that $I^{-}$is closed under $f_{j}$ but $j \in A$. Then $j=r_{k}$ for some $k$. Consider $f_{j}\left(\alpha_{k}\right)$. This cannot be $\beta_{k}$, so we must have $f_{j}\left(\alpha_{k}\right)=\alpha_{k}$, hence $b_{k}\left(\alpha_{k}\right)=s_{k}$ is neither $2 j+1$ nor $2 j+2$, which contradicts the definition of $\alpha_{k}$.

For the converse let $j \notin A$. Pick $x<I^{-}$and write

$$
x=b_{0}(x)+b_{1}(x) c+b_{2}(x) c^{2}+\ldots+b_{c}(x) c^{c},
$$

as above. Pick also $e$ with $x \leq \alpha_{e}$. Then

$$
b_{c}(x) \leq s_{0}, \quad b_{c-1}(x) \leq s_{1}, \quad \ldots, \quad b_{c-e}(x) \leq s_{e} .
$$

CASE 1: $k<c-e$. Then in the expansion $(*)$ there are more than $e$ terms. But $x \leq \alpha_{e}$, so there exists $u \leq e$ such that $b_{c-u}(x)<s_{u}$. Then $f_{j}(x) \leq x+c^{k(x)} \leq \alpha_{e}$ because the exponent $k(x)$ is strictly smaller than $c-u$.

CASE 2: $k>c-e$. Then the expansion $(*)$ of $x$ is shorter than that of $\alpha_{e}$, which contradicts the inequality $x \leq \alpha_{e}$, by $(* *)$.

CASE 3: $k=c-e$. If there exists $u \leq e$ with $b_{c-u}(x)<s_{u}$ then exactly as in case 1 we have $f_{j}(x) \leq x+c^{k} \leq \alpha_{e}$ because $k \leq c-e$. Otherwise we 
have $x=\alpha_{e}$. Then $f_{j}(x)=x$, for otherwise $b_{c-e}=s_{e}$ is either $2 j+1$ or $2 j+2$, so $j$ is of the form $r_{k}$, so it is in $A$, contrary to the assumption.

Observe that the functions $f_{j}$ are not nondecreasing. But the functions $F_{j}(x)=\max \left\{f_{j}(y): y \leq x\right\}$ are nondecreasing and have the same property: $j \notin A$ iff $I^{-}$is closed under $F_{j}$. Granted this we see that $\mathfrak{F}$ cannot be coded in $M$, for otherwise $A=\left\{j \in \mathbb{N}: F_{j} \in \mathfrak{F}\right\}$ would be in $\operatorname{SSy}(M)$ as well.

6. More on the smallest interstice. In this section we do not assume that $M$ is recursively saturated: it is just a model of PA. Our goal here is to give some information about the smallest interstice, $\Omega_{\text {smallest }}=I_{0}(M) \backslash \mathbb{N}$, where, as usual, $I_{0}(M)$ denotes $\inf \{b \in M: b$ is definable and nonstandard $\}$.

Let us point out first that for many models $M$ of PA, $I_{0}(M)=\mathbb{N}$. The quickest way to construct such a model is to use the arithmetized completeness theorem to $\mathbb{N}$ and any completion of PA. A slightly more involved argument exhibits recursively saturated $M$ with this property. On the other hand, for many models $M, I_{0}(M)>\mathbb{N}$. This happens always if $M$ is arithmetically saturated.

It is easy to check that we always have $\mathbb{N} \prec \Sigma_{2} I_{0}(M)$. Moreover, in some respects, $I_{0}(M)$ behaves like a model of $\operatorname{Th}(\mathbb{N})$, e.g., if $M$ is recursively saturated, then every nonstandard element of $I_{0}(M)$ may be moved by an automorphism. In particular, if $I_{0}(M) \models$ PA then $I_{0}(M) \succ \mathbb{N}$.

One of the questions here is whether we have more elementarity. The following construction shows that this need not be the case.

Let $K$ be a model obtained from $M$ by applying the arithmetized completeness theorem, so that the complete diagram of $K$ is arithmetical. To be more specific, we pick a formula $C(\cdot)$ describing a complete extension of PA in $\mathbb{N}$ and put $K=\operatorname{ACT}(\mathbb{N} ; C)$, the model constructed from $\mathbb{N}$ and $C$ by means of the arithmetized completeness theorem. We extend $K$ cofinally by adding an element below all of its nonstandard elements. Working still in $\mathbb{N}$ we add to the language $L_{\mathrm{PA}}$ in the sense of $K$ a new constant $c$ and consider the theory

$$
C \cup\left\{c>t: \exists n C\left(t=S^{n} 0\right)\right\} \cup\left\{c<t: \forall n C\left(t>S^{n} 0\right)\right\} .
$$

This theory is defined inside $\mathbb{N}$ and clearly it is consistent, so it has an arithmetical model, say $R$. $R$ is arithmetical, so $I_{0}(R)$ does not satisfy $\operatorname{Th}(\mathbb{N})$. Indeed, $\operatorname{SSy}\left(I_{0}(R)\right)=\operatorname{SSy}(R)$, and if $I_{0}(R) \models \operatorname{Th}(\mathbb{N})$ then every arithmetical (i.e., definable in $\mathbb{N}$ ) set must be in its standard system. The $R$ constructed above is not recursively saturated. But it is easy to refine this construction to obtain a recursively saturated $R$ with the above properties. Working in $\mathbb{N}$ one extends the language not only by adding the constant $c$ as above, but also a unary symbol $D$ for a satisfaction class. That is, we find (inside $\mathbb{N}$ ) a 
complete extension of the set

$$
\begin{aligned}
\{c>t & \left.: \exists n C\left(t=S^{n} 0\right)\right\} \cup\left\{c<t: \forall n C\left(t>S^{n} 0\right)\right\} \\
& \cup\left\{\operatorname{Sat}(t, D): \exists n C\left(t=S^{n} 0\right)\right\} \cup \text { induction for formulas of } L_{\mathrm{PA}}(c, D) \\
& \cup\left\{\forall \varphi<t[C(\varphi) \rightarrow D(\varphi)]: \exists n D\left(t=S^{n} 0\right)\right\},
\end{aligned}
$$

where $\operatorname{Sat}(e, D)$ expresses " $D$ is a satisfaction class for $Q_{e}$ formulas". Summing up we obtained

Proposition 6.1. There exists a countable recursively saturated model $R$ of PA such that $I_{0}(R)$ does not satisfy $\operatorname{Th}(\mathbb{N})$.

Our goal is to show that models $R$ such that $I_{0}(R) \models \operatorname{Th}(\mathbb{N})$ exist as well. Indeed, we show that if $R$ codes all arithmetical sets by definable elements then $I_{0}(R) \models \operatorname{Th}(\mathbb{N})$. But first let us point out that every countable Scott set is the standard system of some pointwise definable model of PA (see Scott [22]). Kaye [7] contains a detailed exposition of Scott sets. In particular, there exist models $K$ of PA which have the property mentioned above, i.e., $K$ is arithmetically saturated and every arithmetical set is coded by a definable element of $K$. Indeed, if $\mathcal{A}$ is any given Scott set and $M$ is a pointwise definable model with $\operatorname{SSy}(M)=\mathcal{A}$ then we may extend it elementarily to an arithmetically saturated one.

We begin with a construction of an indicator to be used in the sequel. First we write down a formula $A(n, b, c, w)$ which expresses " $b$ is a complete and immediately consistent set of substitutions of the form $\varphi\left(S^{u_{0}} 0, \ldots, S^{u_{m-1}} 0\right)$, where $\varphi \leq n$ and $u_{0}, \ldots, u_{m-1} \leq c$, and each substitution which is in $w$ is in $b$ ". In order to construct this formula, let us say that a substitution of the form $\varphi\left(S^{u_{0}} 0, \ldots, S^{u_{m-1}} 0\right)$ is $n, c$-admissible if $\varphi \leq n$ and $u_{0}, \ldots, u_{m-1} \leq c$. Then $A(n, b, c, w)$ is an abbreviation for the conjunction of:

1. For all $x \in b, x$ is a sentence and there exist $\varphi \leq n$ and $u_{0}, \ldots, u_{m-1} \leq$ $c$ such that $x=\varphi\left(S^{u_{0}} 0, \ldots, S^{u_{m-1}} 0\right)$.

2. For any $\varphi, u_{0}, \ldots, u_{m-1}$, if the substitution $\neg \varphi\left(S^{u_{0}} 0, \ldots, S^{u_{m-1}} 0\right)$ is $n, c$-admissible then exactly one of $\varphi\left(S^{u_{0}} 0, \ldots, S^{u_{m-1}} 0\right), \neg \varphi\left(S^{u_{0}} 0, \ldots\right.$ $\left.\ldots, S^{u_{m-1}} 0\right)$ is in $b$.

3. For every $\varphi, \psi$ if the conjunction $\varphi \& \psi$ is $n, c$-admissible, then it is in $b$ iff both conjuncts are in $b$.

4. For every sentence $\varphi\left(S^{u} 0\right) \in b$ and every $m$, if the sentence $\exists v_{m} \varphi\left(v_{m}\right)$ is $n, c$-admissible then it is in $b$.

5 . For every $x, y, z$, if the statement $S^{x} 0+S^{y} 0=S^{z} 0$ is $n, c$-admissible then it is in $b$ iff $x+y=z$.

6 . The same for other atomic formulas.

7. For every sentence $\varphi \in w$, if $\varphi$ is $n, c$-admissible then it is in $b$. 
The idea here is the following. If $A(n, b, c, w)$ then $b$ is a candidate for truth in the cut $(<c)$ and all statements in $w$ are supposed to be true; $w$ is just a starting point. But the definition gives the quantifier step in one direction, the other direction will be treated below.

Let $B(n, b, c, d, e, w)$ be an abbreviation for the conjunction of the following formulas:

$$
A(n, b, c, w) \& A(n, d, e, w) \& b \subseteq d
$$

and

"for all $\varphi, u_{0}, \ldots, u_{m-1}$ if the statement $\exists v \varphi\left(v, S^{u_{0}} 0, \ldots, S^{u_{m-1}} 0\right)$ is in $b$ then there exists $u$ such that the statement $\varphi\left(S^{u} 0, S^{u_{0}} 0, \ldots, S^{u_{m-1}} 0\right)$ is in $d "$.

We let $\operatorname{Ext}(j, n, \bar{b}, \bar{c}, w)$ be an abbreviation for

$$
\operatorname{Seq}(\bar{b}) \& \operatorname{Seq}(\bar{c}) \& \operatorname{lh}(\bar{b})=\operatorname{lh}(\bar{c})=j \& \bigwedge_{i<j-1} B\left(j, \bar{b}_{i}, \bar{c}_{i}, \bar{b}_{i+1}, \bar{c}_{i+1}, w\right) .
$$

We let $Y(x, y, w)=\max j: \exists \bar{b}, \bar{c}\left[\operatorname{Ext}(j, \bar{b}, \bar{c}, w) \& x \leq \bar{c}_{0} \& \bar{c}_{j-1} \leq y\right]$. It is easy to see that $Y$ is an indicator for the family of cuts satisfying (the standard part of) $w$. It is slightly not obvious that $Y$ is a $\Sigma_{1}$ formula. But $Y(x, y, w)=j$ iff there exist $\bar{b}, \bar{c}$ with

$$
\begin{aligned}
{[\operatorname{Ext}(j, \bar{b}, \bar{c}, w) \& x \leq} & \left.\bar{c}_{0} \& \bar{c}_{j-1} \leq y\right] \\
& \& \neg \exists \overline{b^{\prime}}, \bar{c}^{\prime}\left[\operatorname{Ext}\left(j+1, \bar{b}^{\prime}, \bar{c}^{\prime}, w\right) \& x \leq \bar{c}_{0}^{\prime} \& \bar{c}_{j}^{\prime} \leq y\right] .
\end{aligned}
$$

One may bound the quantifier $\neg \exists$ above by bounding it to the maximum of all sequences of length $\leq y$ with all items $\leq y$ (this gives a bound for $\bar{c}^{\prime}$ ) and binding $\bar{b}^{\prime}$ by the maximum of all sets of sequences (of length $\leq y$ ) of subsets of the interval $(<y)$. This causes no serious difficulty, so we omit the details here.

At the moment we do not assume that $\operatorname{Th}(\mathbb{N}) \in \operatorname{SSy}(M)$. But we let $w_{n}$ be the family of those elements of $T h(\mathbb{N})$ which are $n, n$-admissible. Then $w_{n}$ is in $M$ : indeed, each $w_{n}$ is a finite set of (standard) natural numbers. We also let $Y_{n}(x, y)=Y\left(x, y, w_{n}\right)$. Then each $Y_{n}$ is definable by a $\Sigma_{1}$ formula.

Observe that for every fixed $n \in \mathbb{N}$, there exists a nonstandard $z_{n}$ such that $M=Y_{n}\left(n, z_{n}, z_{n}\right)$. The reason is that for every standard $m, M$ thinks that there exist sequences $\bar{b}, \bar{c}$ which satisfy $\operatorname{Ext}\left(m, \bar{b}, \bar{c}, w_{n}\right)$; one simply takes truth in $\mathbb{N}$ to see this. It follows that either $M$ thinks that this statement holds for each $m$, or the greatest such $m$ is nonstandard.

Let $M=\mathrm{PA}$ be such that $I_{0}(M)>\mathbb{N}$. For every $n \in \mathbb{N}$ we pick a definable nonstandard $z_{n}$ with $Y_{n}\left(n, z_{n}\right) \geq z_{n}$. We choose the appropriate sequences $\bar{b}^{n}, \bar{c}^{n}$. (In this section we use superscripts to distinguish them from subscripts, i.e., indices of the appropriate sequences; we do not need exponentiation.) It will be convenient to have these sequences definable. For 
definiteness, we let $\bar{c}_{0}^{n}=n, \bar{b}_{0}^{n}$ the smallest possible, then $\bar{c}_{1}^{n}$ the smallest possible, etc. This causes no difficulty.

It is easy to see that, when $n$ is fixed, and we are given a cut $I<z_{n}$, then the cut

$$
B^{n}(I)=\sup \left\{\bar{c}_{i}^{n}: i \in I\right\}
$$

has the following property: for every $\varphi \leq n$ and every $u_{0}, \ldots, u_{m-1}$,

$$
B^{n}(I) \models \varphi\left(u_{0}, \ldots, u_{m-1}\right) \quad \text { iff } \quad \exists i \in I M \models \varphi\left(S^{u_{0}} 0, \ldots, S^{u_{m-1}} 0\right) \in \bar{b}_{i}^{n} .
$$

This translation of truth in $B^{n}(I)$ to truth in $M$ is proved immediately by induction on $\varphi$. In fact, the definitions above were given just to ensure this translation.

The apparatus given above (taken from the theory of nonstandard satisfaction classes) will be used to prove the following result. We denote by $\mathrm{SSy}_{0}(M)$ the standard system of the minimal model of $\operatorname{Th}(M)$, i.e., of the Skolem closure of the empty set in $M$.

Theorem 6.2. For every $M \models \mathrm{PA}$, if $\operatorname{Def}(\mathbb{N}) \subseteq \operatorname{SSy}_{0}(M)$ then $I_{0}(M) \models$ $\operatorname{Th}(\mathbb{N})$.

Pr o of. Fix $n \in \mathbb{N}$. Pick a definable element $d$ of $M$ coding truth in $\mathbb{N}$ for formulas $\leq n$. That is, for $u_{0}, \ldots, u_{r-1} \in \mathbb{N}$ and $\varphi \leq n$,

$$
\mathbb{N} \models \varphi\left(u_{0}, \ldots, u_{r-1}\right) \quad \equiv \quad M \models\left\ulcorner\varphi\left(S^{u_{0}} 0, \ldots, S^{u_{r-1}} 0\right)\right\urcorner \in d .
$$

For this $d$ we construct the sequences $\bar{b}, \bar{c}$ in the "natural" manner. That is, we put $\bar{c}_{0}=n$ and $\bar{b}_{0}=d \cap\left(<\bar{b}_{0}\right)$. For each existential statement $\exists u \psi(u)$ which is in $\bar{b}_{0}$ we choose the smallest $u$ such that the statement $\psi\left(S^{u} 0\right)$ is in $d$ and take the maximum of all $u$ obtained in this manner. This maximum is $\bar{c}_{1}$; we also put $\bar{b}_{1}=d \cap\left(<\bar{c}_{1}\right)$. Continuing in the same fashion gives $\bar{b}$ and $\bar{c}$. Observe that $\mathbb{N}$ is closed under the function $\bar{c}_{j} \rightarrow \bar{c}_{j+1}$ and these sequences are of nonstandard length. In particular, their length is greater than $I_{0}(M)$. We claim that

$$
I_{0}(M)=\bigcup_{i \in I_{0}(M)} \bar{c}_{i}^{n} .
$$

First, clearly $i \leq \bar{c}_{i}^{n}$ : indeed, the formula $v_{2}=v_{1}$ ensures this, so the inclusion $\subseteq$ is immediate. For the converse pick $s>I_{0}(M)$. Then for every standard $n, \bar{c}_{n}<s$ because $\mathbb{N}$ and $I_{0}(M)$ are closed under the successor in the sense of $\bar{c}$. It follows that "the greatest $j$ with $\bar{c}_{j}^{n}$ smaller than $s$ " is definable and greater than $\mathbb{N}$, so must be greater than $I_{0}(M)$, so it is not in the right hand side.

By the translation above, the right hand side is the union of a chain which is elementary with respect to formulas $\leq n$, in particular, it satisfies all sentences $\varphi$ which are true in $\mathbb{N}$ and smaller than $n$. Moreover, this holds for every $n$, so $I_{0}(M)$ satisfies the whole $\operatorname{Th}(\mathbb{N})$. 
THEOREM 6.3. If $M$ is a recursively saturated model of PA such that $I_{0}(M)>\mathbb{N}$ and $\operatorname{Th}(\mathbb{N}) \in \operatorname{SSy}_{0}(M)$ then $I_{0}(M)$ is recursively saturated.

Proof. Let $p(x, e)$ be a type over $I_{0}(M)$, in particular the parameter $e$ is in $I_{0}(M)$. Pick a definable $w \in M$ coding $\operatorname{Th}(\mathbb{N})$. For every $n \in \mathbb{N}$ we put $w_{n}=\{\gamma \in w: \gamma \leq n\}$. Construct two sequences $\bar{b}^{n}, \bar{c}^{n}$ exactly as above, the $n$th pair for $w_{n}$. Observe that these sequences are coded in $M$. Exactly as above, $\mathbb{N}$ and $M$ are closed under the successor functions in the sense of $\bar{c}_{n}$. We let $p_{n}(x, e)$ be the subtype of $p(x, e)$ consisting of all elements $\psi(x, e) \in p$ with $\psi \leq n$.

We let $j_{n}=1+\min \left\{i: \bar{c}_{i}^{n}>e\right\}$. Pick also coded sequence $\beta_{k}$ of definable elements of $M$ convergent to $I_{0}(M)$ from above. Then we have

$$
\text { (i) } \bar{c}_{j_{n}}^{n}<\beta_{k} \text { for all } k \text { and (ii) } \exists x<\bar{c}_{j_{n}}^{n}\left\ulcorner\mathbb{M} p_{n}\left(S^{x} 0, S^{e} 0\right)\right\urcorner \in \bar{b}_{j_{m}}^{m} \text {. }
$$

Consider the type

$$
\Gamma(x)=\left\{\left\ulcorner\mathbb{M} p_{n}\left(S^{x} 0, S^{e} 0\right)\right\urcorner \in \bar{b}_{j_{n}}^{n}: n \in \mathbb{N}\right\}
$$

whose parameters are $e$ and sequences coding $\bar{b}^{n}: n \in \mathbb{N}$ and $c_{j_{n}}^{n}: n \in \mathbb{N}$. Obviously, this type is finitely realized, each of its realizations is in $I_{0}(M)$, and by the translation given above, each element realizing $\Gamma$ in $M$ realizes $p$ in $I_{0}(M)$. Also, it is easy to replace infinitely many parameters $\bar{b}^{n}: n \in \mathbb{N}$ and $j_{n}: n \in \mathbb{N}$ by the single parameter $w$.

7. A remark on pseudogaps. In all the results in [1] and above we worked with intersticial gaps defined by means of nondecreasing definable functions. There exists another candidate for this notion. It is as follows. Work with a fixed interstice $\Omega=I^{+} \backslash I^{-}$in $M$. Given $a \in \Omega$ define two cuts $I^{d}$ and $I^{g}$ by $I^{d}=\inf \{t(a): t$ is a term such that $t(a) \in \Omega\}$ and let $I^{g}$ denote the supremum of the same set. Call the space between these two cuts, i.e., $I^{g} \backslash I^{d}$, the pseudogap of $a$. In several proofs, especially in the moving gaps and covering gaps lemmas from [1], it was essential that we worked with nondecreasing functions (i.e., with the ordinary intersticial gaps) rather than with arbitrary definable functions. Of course, the situation would be much clearer if we could prove that the notions of pseudogaps and gaps are the same. Unfortunately, it is not so.

THEOREM 7.1. If $M$ is an arithmetically saturated model of some false completion of PA then there exists a $\in \Omega_{\text {smallest }}$ such that the gap of a is strictly included in the pseudogap of a.

In order to construct the appropriate $a$ we need a function $D$ for which we have no sensible control on inequalities between values of $D$. There is a "natural" candidate for such a function. It is defined as follows. Let $\operatorname{Tr}_{0}$ 
denote, as usual, the universal formula for $\Delta_{0}$ formulas. We let

$$
D(x)=\left\{\begin{array}{l}
\text { the } y \text { such that } \operatorname{Tr}_{0}\left(\varphi\left(S^{u} 0, S^{y} 0\right)\right) \\
\text { if } \exists \varphi, u\left[x=\varphi\left(S^{u} 0, v\right) \& \varphi \in \Delta_{0} \& \exists ! y \operatorname{Tr}_{0}\left(\varphi\left(S^{u} 0, S^{y} 0\right)\right)\right], \\
0 \quad \text { otherwise. }
\end{array}\right.
$$

Recall that by the arithmetical saturation of $M$, the family $\mathfrak{F}$ of nondecreasing definable (in $M$ ) functions under which $\mathbb{N}$ is closed, is coded in $\operatorname{SSy}(M)$, so may be used in types.

Pick $e \in I_{0}(M) \backslash \mathbb{N}$ and consider the type

$$
\begin{array}{r}
\Gamma(x)=\left\{x>e \& \exists \varphi, u\left[\varphi \in \Delta_{0} \& x=\varphi\left(S^{u} 0, v\right) \& \exists ! y \operatorname{Tr}_{0}\left(\varphi\left(S^{u} 0, S^{y} 0\right)\right)\right.\right. \\
\left.\left.\& \exists y\left[n<y<F(x) \& \operatorname{Tr}_{0}\left(\varphi\left(S^{u} 0, S^{y} 0\right)\right)\right]\right]: n \in \mathbb{N}, F \in \mathfrak{F}\right\} .
\end{array}
$$

Observe that if $x$ realizes $\Gamma$ then the appropriate $\varphi\left(S^{u} 0, v\right)$ defines (in the sense of $\operatorname{Tr}_{0}$ ) an element $y$ which is in the pseudogap around $x$ but not in the gap around $x$. Hence, it suffices to check that $\Gamma$ is consistent. But this is easy. Indeed, if $\Gamma_{0}$ is a finite part of $\Gamma$ then $x$ of the form $\left\ulcorner v=S^{r} 0 \& \mathbb{A}_{i<e} \varrho_{i}\right\urcorner$ (where each $\varrho_{i}$ is $0=0$ ) realizes $\Gamma_{0}$ for $r$ large enough.

Observe that the same argument works for each interstice $\Omega=I^{+} \backslash I^{-}$ such that $I^{-}$is closed under (primitive recursive functions representing) logical operations and at least one of the sequences $\alpha_{n}, \beta_{n}$ as in Lemma 1.3 contains only elements $\Sigma_{k}$-definable for some $k$ which is independent of $n$.

\section{References}

[1] N. Bamber and H. Kotlarski, On interstices in countable arithmetically saturated models of Peano Arithmetic, Math. Logic Quart. 43 (1997), 525-540.

[2] T. Bigorajska and H. Kotlarski, A partition theorem for $\alpha$-large sets, to appear.

[3] P. Erdős and R. Rado, A combinatorial theorem, J. London Math. Soc. 25 (1950), 249-255.

[4] R. Graham, B. Rothschild and J. Spencer, Ramsey Theory, 2nd ed., Wiley, 1990.

[5] A. Grzegorczyk, Some classes of recursive functions, Dissertationes Math. 4 (1953).

[6] P. Hájek and P. Pudlák, Metamathematics of First Order Arithmetic, Perspect. Math. Logic, Springer, 1993.

[7] R. Kaye, Models of Peano Arithmetic, Oxford Logic Guides, Oxford Univ. Press, 1991.

[8] R. Kaye, R. Kossak and H. Kotlarski, Automorphisms of recursively saturated models of arithmetic, Ann. Pure Appl. Logic 55 (1991), 67-91.

[9] J. Ketonen and R. Solovay, Rapidly growing Ramsey functions, Ann. of Math. 113 (1981), 267-314.

[10] R. Kossak, H. Kotlarski and J. Schmerl, On maximal subgroups of the automorphism group of a countable recursively saturated model of PA, Ann. Pure Appl. Logic 65 (1993), 125-148. 
[11] R. Kossak and J. Schmerl, The automorphism group of an arithmetically saturated model of Peano arithmetic, J. London Math. Soc. (2) 52 (1995), 235-244.

[12] - - - Arithmetically saturated models of arithmetic, Notre Dame J. Formal Logic 36 (1995), 531-546.

[13] H. Kotlarski, On elementary cuts in recursively saturated models of arithmetic, Fund. Math. 120 (1984), 205-222.

[14] - , Automorphisms of countable recursively saturated models of PA: a survey, Notre Dame J. Formal Logic 36 (1995), 505-518.

[15] H. Kotlarski and R. Kaye, Automorphisms of models of True Arithmetic: recognising some basic open subgroups, ibid. 35 (1994), 1-14.

[16] H. Kotlarski and Z. Ratajczyk, Inductive full satisfaction classes, Ann. Pure Appl. Logic 47 (1990), 199-223.

[17] - - - More on induction in the language with a satisfaction class, Z. Math. Logik 36 (1990), 441-454.

[18] W. Pohlers, Proof Theory, Lecture Notes in Math. 1047, Springer, 1989.

[19] Z. Ratajczyk, A combinatorial analysis of functions provably recursive in $I \Sigma_{n}$, Fund. Math. 130 (1988), 191-213.

[20] - Subsystems of true arithmetic and hierarchies of functions, Ann. Pure Appl. Logic 64 (1993), 95-152.

[21] H. Rogers, The Theory of Recursive Functions and Effective Computability, McGraw-Hill, 1967.

[22] D. Scott, Algebras of sets binumerable in complete extensions of arithmetic, in: Recursive Function Theory, Amer. Math. Soc., Providence, 1962, 117-121.

[23] R. Sommer, Transfinite induction within Peano arithmetic, Ann. Pure Appl. Logic 76 (1995), 231-289.

Institute of Mathematics

Agricultural and Pedagogical University

Orlicz-Dreszera 19/21

08-110 Siedlce, Poland

E-mail: terebi@wsrp.siedlce.pl

hkl@impan.gov.pl
Department of Mathematics University of Connecticut Storrs, Connecticut 06269

U.S.A.

E-mail: schmerl@math.uconn.edu 